\title{
Of Syringes, Vaccines and Chemistry
}

\author{
Benjamín Ruiz Loyola* and Jorge Benjamín Ruiz Gutiérrez \\ Department of Organic Chemistry, Mexico
}

*Corresponding author: Benjamín Ruiz Loyola, Department of Organic Chemistry, UNAM, Mexico

Received: 柴 June 03, 2019

Published: 眥June 14, 2019

\begin{abstract}
The modern trend to avoid vaccination is based mainly on superstición and bad science understanding and includes a bad medical practice. In this article we talk about how vaccines were originated, if the syringe development and the importance of materials chemistry to improve its manufacture.
\end{abstract}

Keywords: Vaccine; Syringe; Immunization; Jenner; Wood

\section{The Beginnings of Immunization}

Since its appearance on the face of the planet, the human being has been accompanied by diseases that cause infections and that are easily transmitted from person to person. This is logical if we think that the first living beings were the unicellular ones; thus, it is not surprising that epidemics were already mentioned before our era and, in addition, possible ways of surviving the infection. It is possible that in India, 1,500 B. C., epidemics and forms of a very early vaccination were documented. It is mentioned that the Egyptians sought some form of inoculation with microorganisms, while Thucydides refers to some plague saying: “... this disease is not suffered twice and if it were to suffer again, it would not be with deadly results ..., which leads to think that non-fatal infected people were immunized, similar to what happens with vaccines. From shortly before the beginning of the Christian era and until the twelfth century, the Chinese used to dry the pustules of both humans and cows infected with smallpox. Once dry, they sprayed them and made people, especially those who had daily contact with cattle, inhale the dust through their noses to try to get immunized, which was achieved in a huge number of cases. This technique was called scarification. Another practice that was developed in the search for control of these pests was the use of material infected with smallpox among humans: food was prepared, for example, and the sickest person was the first one to eat it, to later pass it in the same recipient among all the members of the family or community [1].

Many of these customs persisted in the Middle East and were introduced in Europe mainly by the Ottoman invasions; but in the eighteenth century the wife of the English ambassador in
Turkey (who had applied the technique to her own six-year-old son) proposed the use of scarification to deal with the smallpox epidemic that struck London in 1721. Applied experimentally about some inmates and in view of the positive results, the princesses Amelia and Carolina, daughters of the Prince of Wales and future King George II were subjected to this treatment; they suffered the disease very slightly and had no sequelae or the horrible marks left on the faces of the survivors. The scarification gained ground until Edward Jenner, an English physician, developed a different and properly protocolized method. A milkmaid who had lesions with pus on her hands (presumably cowpox), named Sarah Nelmes, allowed Jenner to take that pus and with it she inoculated a child, James Phipps. The inoculation was usually done with a metal lancet that was put in contact with the infected material, to then produce pickets or small cuts in the skin of the people to whom it was wished to infect. We must emphasize that before experimenting with the child Phipps, Jenner had inoculated his own son of 18 months of age. Six weeks after this inoculation James was inoculated again, now with pus from active lesions of human pox. The child Phipps did not get infected and Jenner waited a month to try again to infect him with human smallpox. Excited by this success, Jenner repeated the experiment with 32 more people, achieving the immunity of all of them [2]. Despite all the setbacks that occurred, Jenner went ahead and in 1803 the entire royal family was vaccinated with his technique, which began to be used in Europe and then around the world. Perhaps the memory that George Washington was a survivor of smallpox, led to the acceptance of the method in the United States. It is said that the first Russian child vaccinated was called Vaccinov (because of the vaccine). On the other hand, Napoleón I in 
1812 decreed obligatory the vaccination against the smallpox in the army and tried to take it to all the French society [3].

\section{The Beginning of the Injection}

Ovid tells that Medea helped Jason get the golden fleece in exchange for his promise of marriage. Medea was a powerful priestess and sorceress who returned youth to Jason's father, Esón, cutting his throat and then filling his body with a magic potion. He did this with a double purpose: to get well with Jason and demonstrate his power. There are many researchers who assume that the act performed by Medea to rejuvenate Esón was simply a blood transfusion. We would be talking about introducing a foreign substance to the body, more than two thousand years ago. The next historical reference to a blood transfusion is available until the fifteenth century, when blood was taken from three young men -who died for it- for a Jewish doctor to carry out a transfusion to Pope Innocent VIII. However, we do not know anything about the way in which these transfusions were carried out. In the midseventeenth century Christofer Wren, assisted among others by Robert Boyle (the alchemist and scientist who enunciated Boyle's law for gases, among other principles) administered intravenous drugs to patients. A cut was made in the vein, a silver or gold tube was inserted, and the liquid was introduced by pumping it through a leather bladder (like an enema, an enema) or by simple gravity. The medications used were water, opium or purgatives; many of these procedures were aimed at treating syphilis. Towards the nineteenth century an instrument was developed that allowed to pump liquids in small quantities with greater comfort; these were hand-held devices (not as large as enema bladders), which fit in one hand. They consisted of a hollow tube in which a plunger was inserted to push the contents of the tube towards the end; This was also hollow and was introduced in various parts of the body for the administration of substances $[4,5]$.

The wife of the Scottish scientist Alexander Wood suffered from very intense neuralgia and that motivated Word to find a way to alleviate his pain more quickly and effectively. To make the opium action faster and more effective, Wood used some device designs that allowed him to "push" a drug through a kind of pump and managed to improve this design (simultaneously with the work of the French Charles Gabriel Pravaz) by using a needle with a hollow body and tip; with this the medicine could be introduced in the skin of the patient, looking for the rapidity in the administration. Wood argued that, at least in the case of analgesics such as morphine (opium), it should be deposited in a nerve very close to the site in which the pain was located. He published an article about these studies entitled A new method to treat neuralgia through the direct application of opioids in pain points. A legend says that Wood's excitement at experimenting on how to cure pain caused him to administer too many injections in a short time, until his wife died, but without pain. This has not been proven historically, but it is still a curious detail, not forgetting the fact that what Wood's wife really had was a kind of arthritis. Another form of this legend says that it was about migraines, but the article written by Wood is quite clear and points out the application in painful joints. The important thing of this matter is that a way of depositing the opiates was found in such a way that if they caused side effects on the digestive system (esophagus, stomach or intestines), they no longer appeared. Obviously, the first deduction was that if they were deposited near the affected sites, the opioids would work better. For five years this was applied by those doctors who believed in this novel technique, until Dr. Charles Hunter was presented with a complication: two of his patients developed local abscesses precisely at the sites where they received injections. This caused Hunter to experiment to see if the same response could be obtained by depositing the analgesics away from the directly affected sites and the results were positive. Hunter coined the term hypodermic (under the skin) but had to face a demand established by Wood accusing him of plagiarism and malpractice. The medical journals became battlefields until a committee of the Surgical Medical Society of London was formed, which two years later ruled in favor of Hunter: the new technique would be called hypodermic and it was concluded that it was possible that the injection of opiates will be successfully managed away from the site of pain. The most curious of all this is that no one, neither the doctors involved with Wood nor those who were in favor of Hunter, nor the Medical Society itself thought about the problems that were causing the patients: tolerance, addiction and over demand, among others, for how lucrative it was to have so many patients and so much opium to administer. Be that as it may, the hollow needle and the hypodermic syringe, the predecessor of the one we use today to administer medicines and vaccines or to draw blood, for example, were invented and perfected. Or to fill ink cartridges for printer.

\section{Materials of Construction of Syringes}

Initially the plunger or piston of the syringes had cotton on the tip so that there was a good fit between the body of the syringe and the piston, which allowed the liquid to be driven. The end of the syringe was conical in shape and the hollow needle was adapted at the end. It was initially worked with metals but soon changed to glass, because of its quality of not reacting with the substances that were injected. For example, morphine is obtained from opium using hydrochloric acid and consequently the liquid that was injected to the patients had acidic characteristics, which could react with the metal. To not break the glass easily and give greater security to those who used these syringes, the body of these was inserted into a metal support spice that allowed rapid handling while showing the amount of substance that was injected. The twentieth century brought several changes in the design and construction of syringes. The common glass is known as alkaline glass and, over time, becomes opaque by washing and contact with water and other materials. To eliminate this problem, we began to use alkali-free glass (Pyrex type); later, a stainless-steel tip attached to the glass, which allowed to secure the needle so that it would not slip and accidentally separate when applying the injection was designed. Thus, the syringes came in stainless steel cases that were also used to sterilize the syringe boiling water in the case itself, which closed hermetically to maintain the sterility of the instrument. The Second World War allowed the development of an auto injection device that would allow the soldiers to inject analgesics or specific antidotes while they were attended by doctors. The innovation was that the needle remained hidden from view by the user, reducing the anxiety 
that causes most people to know that they are going to inject it. The syringe consisted of a tube filled with the specific medication to be used and a spring that, when hit against a member of the soldier, pushed the needle towards the site of the blow, causing it to penetrate under the skin and release its contents. Towards the middle of the century, the first fully disposable glass syringes were produced with two different purposes and designs: one to collect blood in donation campaigns and the other for polio vaccination campaigns. Meanwhile the steel for the needles and the specific cut for each application were perfected: it was no longer just a question of perforating the skin, but the angle determined whether it was a subcutaneous, intramuscular or intravenous injection. They also worked on finding a way to make disposable needles. The 1960's decade not only brought the Beatles to the world; they also brought the completely disposable syringes made of plastic and that were marketed in previously sterilized packages.

\section{Encounter between Hypodermal Syringes and Vaccines}

From the development of the hypodermic syringe and the hollow needle, the application of the vaccines could be carried out more efficiently, measuring the quantities that were introduced to the body with greater precision. Pasteur's experiment to find a vaccine against anthrax in cattle is famous, but in the field of human vaccines it is also at least remarkably successful. In 5 , without being a doctor and therefore not authorized to give any treatment to any person, Pasteur decided to use the vaccine against rabies developed by his colleague Emile Roux drying the spinal cord of rabbits infected with the disease to treat the child Joseph Meister, who had been attacked by a rabid dog. The child did not develop the disease and Pasteur remained a hero, although if the vaccine had failed, he might have been condemned for his audacity. The important part is that this vaccine was not inoculated but injected. From then on, most vaccines are applied by injection, although many are already ingested.

\section{Vaccine Production}

There are several different types of vaccines, depending on how the antigens are produced, that is, the microorganisms or chemicals responsible for unleashing the body's immune response. Let's see them quickly.

\section{Living Attenuated Microorganisms}

They consist of using the weakened (attenuated) microorganism, which is the closest thing to a real infection. The advantage is that it is the infectious agent itself that provides the immunity; The disadvantages are the need for refrigeration and the possibility of mutations, among others. This form of vaccine is very suitable for the case of viruses. The vaccines against rubella, varicella and some types of influenza, among others, correspond to this kind of preparation.

\section{Inactivated Microorganisms}

Dead microorganisms are used; This procedure is better for bacteria. In the case of using this type of vaccines, we have the disadvantage that, as the bodys immune response is slower and slower, it requires the use of reinforcements, that is, new applications of the vaccine. Among the advantages of this kind of vaccine are its stability and its ease of transport and storage, since they do not require refrigeration. Some of the vaccines in this class are rabies, hepatitis $\mathrm{A}$, some types of influenza and polio.

\section{Vaccines in Subunits (Parts of Viruses)}

Instead of using the entire microorganism, only the parts that the antibodies recognize to affect their immunizing action are used. This technique greatly reduces the possibility of adverse side reactions occurring in vaccinated persons. Usually cultures of the microorganism are prepared to then use chemical techniques to separate the specific part that leads to obtain the vaccine. Genetic engineering techniques such as recombinant DNA technology can also be used to produce this vaccine. This is how some vaccines against hepatitis $\mathrm{B}$ and human papillomavirus are prepared and research is under way to reach the hepatitis $C$ vaccine. They have the advantage that they target a part of the microorganism specifically, and that there are less chances of adverse reactions, but in counterpart its development is more difficult and takes more time.

\section{Toxoids}

When a microorganism secretes a toxin (toxic chemical compound in extremely low doses) that causes the disease, the microorganism is cultured to obtain appreciable amounts of the toxin. The microorganism is separated, the toxin is purified and rendered inactive, usually by using a solution of formaldehyde in sterilized water. These detoxified toxins are used as vaccines; When the human body receives this chemical compound deactivated, it learns to fight it and prepares itself for an eventual confrontation with the original toxin. Examples of this class of vaccine are diphtheria and tetanus. Vaccines also contain other chemical compounds as ingredients to make them safer and more effective. Among the most important we have the following.

\section{Preservatives}

Prevent the contamination of containers, bottles or culture media with pathogenic germs that could cause other diseases than those prevented by the vaccine. One of the most used preservatives in the past was the so-called thimerosal, a compound that contains mercury. Its use was restricted to the production steps and was eliminated when the vaccine was packaged for storage and transport. Currently, most of the vaccines have been eliminated, but they are still used in some processes, such as some influenza vaccines.

\section{Additives}

They help the vaccine retain its potency during storage time; Among other chemical substances used for this purpose, we have gelatin, albumin, sucrose and lactose.

\section{Adjuvants}

They help the body to have a better immune response and are usually aluminum salts. These salts help the antigens remain in the injection site and then be transported directly to the lymph 
nodes, which is where the immune response to the presence of the aggressors begins.

\section{Antibiotics}

Sometimes antibiotics can be used to prevent contamination of vaccines. In very isolated cases traces of antibiotics present in vaccines can trigger allergic reactions in vaccinated subjects. Some vaccines are produced in chicken eggs, so that some proteins from the same egg may be present in the vaccine and cause allergic reactions; vaccines against influenza and against yellow fever are produced in this way. Each vaccine requires very specific and controlled conditions for its production: the mixtures of compounds that will serve to cultivate the microorganism, those of the substances that will allow its isolation and conservation; in short, without the achievements of chemistry, we would not have the spectacular advances that allow a huge number of diseases that were deadly a few decades ago can now be prevented. As we can see, chemistry has provided materials so that we can prevent or cure diseases, making our life longer and more pleasant.

\section{References}

1. Stanley A Plotkin (2005) Vaccines: Past, present and future. Nature Medicine Supplement 11(4): S5-S11.

2. Lloyd J (2000) Technologies for vaccine delivery in the $21^{\text {st }}$ entury, World Health Organization. Depatment of vaccines and biologicals, Geneva, Switzerland.

3. Phil Ball, Crawford Brown, Karolin Lindström, (2009) 21 $1^{\text {st }}$ Century Vaccine Manufacture. BioProcess International p. 18-28.

4. Jaw Wildsmith (2012) Some (mostly Scottish) local anaesthetic héroes. J R Physicians Edimb 42: 179-83.

5. Hardt K, Schmidt-Ott R, Glisman S, Adegbola R A, Meurice FP, et al. (2013) Sustaining Vaccine Confidence in the $21^{\text {st }}$ Century. Vaccines $1(3)$ : 204-224.

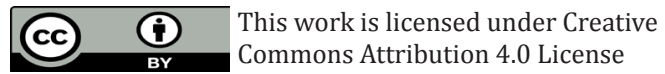

To Submit Your Article Click Here:

\section{Submit Article}

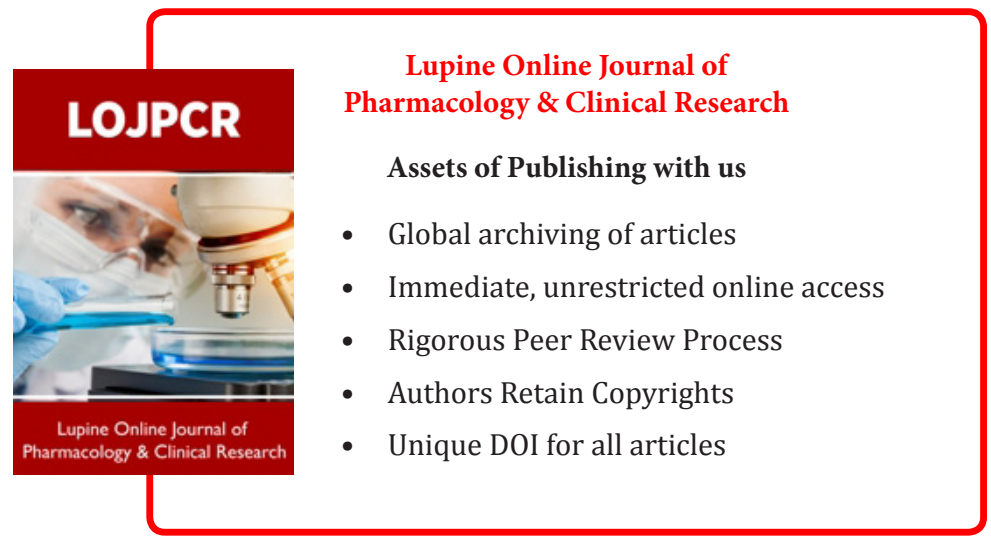

